\title{
A Survey: Sparse Traffic Grooming and RWA Assignment Schemes in Optical Network
}

\author{
A. S. Shinde \\ Department of Information Technology \\ Sinhgad Academy of Engineering, Pune
}

\author{
M. R. Dhage \\ Computer Engineering Department \\ Sinhgad College of Engineering, Pune
}

\begin{abstract}
Traffic Grooming has become a very important issue on optical Network, as optical networks provide a very high speed data transmission for huge amount of data. A Sparse grooming Network with only a fractional of nodes having grooming functionalities may achieve the same performance as the one in which all the nodes are grooming, but with much lower cost. In literature different algorithms, models and techniques have been proposed to design the sparse grooming networks. With Proper assignment of routing and wavelengths in the network reduces the blocking probability ultimately increases the bandwidth of the network. In this paper, we studied and analyzed the different sparse traffic grooming and RWA assignment strategies with its performance metrics for optical mesh networks.
\end{abstract}

\section{Keywords}

OXC - Optical Cross Connect, OC-Optical carrier, RWARouting \& wavelength assignment, WDM - wavelength division multiplexing, G-Fabric - Grooming Fabric, G-Node Grooming Node, G-OXC - Grooming Optical Cross Connect, W-Fabric - wavelength-switching fabric.

\section{INTRODUCTION}

The backbone network is mostly a mesh network with optical fibers. Optical fiber technology gives very high speed transmission speed because of its potentially limitless capabilities, a huge bandwidth (over 50 terabits per second (Tbps)). On the other hand, only a fraction of customers are expected to have a need for such a high bandwidth. Due to large cost of the optical backbone infrastructure it is necessary to combine low-speed traffic streams onto high-speed mesh network not all nodes need to have grooming capabilities. A network with only a fraction of nodes having grooming functionalities, called as a Sparse Grooming network. A node which has traffic -grooming capability is called as grooming node (G-Node). Hence, the problem of designing a sparsegrooming WDM mesh network is a very important and practical problem.

Figure 1 shows a sample G-OXC architecture. There are two switching fabrics in this $\mathrm{OXC}$, a wavelength-switching fabric (W-Fabric) and a grooming fabric (G-Fabric). Because a grooming OXC may be more costly than an OXC without grooming capability (i.e., the OXCs which only have the WFabric), and in some optical WDM mesh network, only a few network nodes may have traffic-grooming capability. This type of network a "sparse-grooming network", and a node which has traffic-grooming capability to be a grooming node (G-Node)

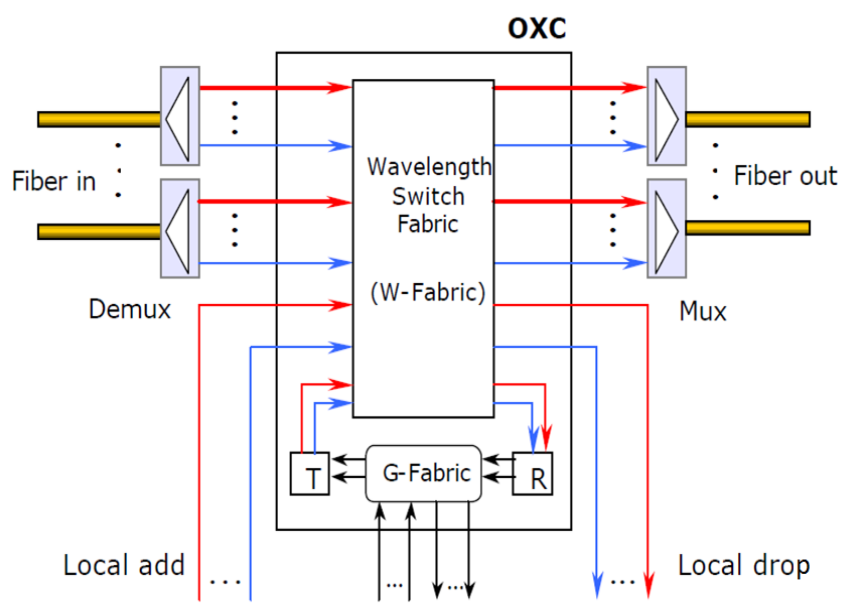

Figure 1 : An OXC with a two-level hierarchy and grooming capability.

Once the sparse grooming network is designed from given physical optical backbone network, i.e. it forms a network with some nodes to be designated as grooming nodes. The grooming nodes along with the non-grooming nodes form a logical or virtual topology of the backbone network. Based on the arrival of call or traffic request the light-paths are established between source and destination nodes. The light paths established are based on the k-shortest path routing, and depending on the availability of wavelength channels. To establish a lightpath in a WDM network, it is necessary to determine the route over which the lightpath should be established and the wavelength to be used on all the links along the route. This problem is called the routing and wavelength assignment (RWA) problem.

Most of the previous research focuses on some routing and wavelength assignment schemes, which are summarized below.

\subsection{Routing}

The current used routing approaches are fixed routing, fixedalternate routing, and adaptive routing.

\subsubsection{Fixed routing}

In fixed routing, the connections are always routed through a predefined fixed route for a given source-destination pair. If there are not enough resources to satisfy a connection request, the connection gets blocked.

\subsubsection{Fixed-alternate routing}

In fixed-alternate routing, multiple fixed routes are considered when a connection request comes. In this approach, each node in the network is required to maintain a routing table that 
contains an ordered list of a number of fixed routes to each destination node. It is simple as compared with other and widely used in the dynamic connection provisioning case.

\subsubsection{Adaptive routing}

In adaptive routing, the route from a source node to a destination node is chosen dynamically, depending on the current network state. Adaptive routing will require more computation and a longer setup time than fixed-alternate routing, but it is also more flexible than fixed- alternate routing.

\subsection{Wavelength Assignment schemes}

Wavelength Assignment is the main factor that affects the blocking probability and thereby the performance of the network. Proper assignment of wavelengths can lead to reduced or no use of wavelength converters which can significantly reduce the cost. As mentioned above, wavelength assignment can be broadly classified into two categories, static (off-line) and dynamic (on-line) assignment. In a static assignment scheme the same wavelength is assigned, if available, for every lightpath request generated at a node or else the request is blocked. In dynamic allocation the node uses a following algorithm that selects a particular free wavelength at that node, if available, and then assigns it to that request and routes it, otherwise the request is blocked. Most of the assignment strategies available in the literature are dynamic in nature. The algorithm for every assignment scheme maintains a list of used and free wavelengths at each node. Whenever a call is generated the node selects a wavelength from the set of free wavelengths and assigns it to that call. Some of the proposed wavelength-assignment algorithms are as follows:

\subsubsection{Random wavelength assignment}

In given topology, the node maintains a list of free wavelengths at every moment, whenever a call is arrived the node randomly chooses a any wavelength from the set of free wavelengths and assigns that wavelength to that call.

\subsubsection{First-fit wavelength assignment}

This strategy maintains the predefine order on the wavelengths; it maintains a list of used and free wavelengths. The assignment scheme always chooses the lowest indexed wavelength from the list of free wavelengths and assigns it to the request.

\subsubsection{Most-used wavelength assignment}

It uses the free wavelength that is used for maximum times in the network and assigned to called request.

The Problem with Most-used wavelength assignment: Firstly, it requires the entire knowledge of the network.

\subsubsection{Least-used wavelength assignment}

It is similar to the most-used wavelength strategy, but in this strategy the least used wavelength in the network is assigned. The main purpose of this approach is to achieve a nearuniform distribution of the load over the wavelength set.

\section{LITERATURE SURVEY}

Keyao Zhu et. al. [1] has investigated the problem of designing a sparse-grooming WDM mesh network with static traffic. As with proper design of sparse network, increases the network throughput. In the design part some selected nodes do grooming functionality hence ultimately it reduces the overall network cost also. Previously in the literature, it is assumed that every network node has traffic-grooming capability, which may not be practical or cost-effective solution in a WDM backbone network. The problem is mathematically formulated and the proposed a heuristic approach which compares the network throughput with the number of grooming nodes selected from the given network. The groom nodes are selected on the basis of three criterions which are described below.

\section{$>$ Nodal degree selection \\ $>$ Amount of bypass traffic \\ $>$ Random selection.}

Nodal-Degree Selection: The nodal degree of a node in a network is the number of connections the node has to other nodes. In this scheme, the nodes which have the maximum nodal degree are picked to be G-Nodes. If several nodes have same nodal degree and only some of them can be chosen, random selection is used to break any ties.

Bypass-Traffic Selection: In this scheme it selects a $\mathrm{G}$ nodes based on, the node which bypasses maximum amount of traffic. This will be analyzed by counting the number of call blocks on each node. And finally the nodes which have maximum blocked calls can be selected as the G-Nodes.

Random Selection: In this scheme, the nodes are randomly picked to be G-Nodes.

Wang Yao et. al.[2] had proposed the maximize-lightpath sharing multi-hop (MLS-MH) grooming algorithm to provision dynamically arriving multi-granularity connections in sparse grooming networks. This model support dynamic traffic grooming in sparse grooming networks. They have evaluate the performance in terms of call blocked in the network.

Partha Paul et. al.[3] proposed a heuristic approach using maxconnectivity grooming for solving GRWA problem with dynamic traffic requests. As their results prove that maxconnectivity grooming uses minimal number of grooming devices and thus effectively reduces the network cost compared to other grooming schemes. The system also focus on the formulation of grooming, routing and wavelength assignment (GRWA) problem in WDM mesh networks with dynamic traffic under the constraints of the number of grooming devices used and wavelength continuity. It uses first fit wavelength assignment procedure.

Osama Awwad et. al.[7] had proposed two novel Heuristics approach that minimize the cost of the traffic grooming and wavelength conversion equipment used in optical network without disturbing the network blocking performance. As this approach focus on both traffic grooming with RWA problem hence it named as GRWA problem, previously in literature was shown that the problem of traffic grooming, routing and wavelength assignment handles separately ,but genetic approach described by author handle it combine. The grooming problem can be performed at single-hop the performance was evaluated in terms of the total cost of traffic grooming and wavelength conversion devices used of the network.

Jun Zhou et.al. [8] investigate the impact of non accurate state information on the performance of dynamic routing and wavelength assignment (RWA) algorithms. This study shows that effective RWA algorithm must be able to tolerate the imprecise global network state information and make effective routing and wavelength assignment decisions in the presence of imprecise global network state information. They have assumed a timer-based link state update scheme which updates link state periodically with a given time interval. The 
main objective of a dynamic routing and wavelength assignment algorithm is to select a path and a wavelength on each link along the path such that the blocking probability is minimized. As this study focus on two types of network called WS (Wavelength-selective) in such a network, a connection can only be established if the same wavelength is available on all links between the source and the destination. Another type is WI (wavelength-interchangeable) networks which allow to change the wavelength from source to destination path. With respect to the WI network it has three different wavelength assignment algorithms like first, most and best fit. This study shows that the performance of network in terms of blocking probability with respect to the traffic load by selecting appropriate wavelength assignment scheme.

Ashok Kumar Pradhan et. al.[9] has addressed multicast traffic grooming problem to maximize the bandwidth in optical network. Proposed heuristic approach Prioritized multicast traffic grooming (PMTG) was used to reduce maximum number of wavelengths in optical network, only when grooming of incoming request call performed successfully.

Result have proved that PMTG gives better performance than Multicast traffic grooming with shortest path algorithm( MTG-SP) .The performance was measured in terms of wavelength required for any connection request.

Amrinder S. Arora et. al.[10] addressed the problem of optimally placing a limited number of wavelength converter in WDM optical Mesh network. A new heuristic algorithm proposed for the placement of wavelength converters ultimately minimizes the average blocking probability. The performance of heuristic approach measured using analytical model.

Gangxiang shen et. al.[11] focuses on sub-wavelength traffic grooming in translucent optical network. A Virtual nodal degree ranked algorithm was proposed to select the best place of opaque switch node. Mixed-integer linear programming (MILP) optimization model was developed to groom subwavelength traffic request in network. This model maximizes served sub-wavelength traffic demand and minimizes the required wavelength capacity.

\section{CONCLUSION}

In recent years, most of attention has been given on the Traffic Grooming, Routing and wavelength assignment schemes, as it is very crucial factor of the Optical Network. Many researchers have worked on Sparse Traffic Grooming in order to minimize the Blocking probability of the network ultimately it improves the performance as well. In this paper I have tried to summarize various techniques used for sparse traffic grooming and RWA schemes in optical network.

\section{ACKNOWLEDGEMENT}

I would like to convey thanks to my guide, Prof. Manisha R. Dhage for a number of comments and suggestions to improving this paper.

\section{REFERENCES}

[1] K. Zhu, H. Zang, B. Mukherjee, "Design of WDM Mesh Networks with Sparse Grooming Capability", in Proc. IEEE Globecom 2002, vol.3.pp. 2696-2700, Nov. 2002.
[2] W. Yao, M. Li, and B. Ramamurthy, "Performance analysis of sparse traffic grooming in WDM mesh networks", in Proc. IEEE ICC, 2005, pp. 1766-1770.

[3] Partha Paul, Balbeer. S. Rawat, Swapan K. Ghorai, "Dynamic Traffic Grooming in WDM Optical Networks with Full Wavelength Conversion and Grooming Devices on Max-Connectivity Nodes", International Journal of Computer Applications(0975-8887), Volume 57-No.11, November 2012.

[4] K. Zhu and B. Mukherjee, "Traffic grooming in an optical WDM mesh network", IEEE Journal on Selected Areas in Communications, vol. 20, no. 1, pp. 122133,Jan. 2002.

[5] M. EI Houmaidi, M. A. Bassiouni and G. Li, "Optimal traffic grooming in WDM mesh networks under dynamic traffic", in Optical Fiber Communication Conference, Technical Digest (CD) (Optical Society of America, 2004).

[6] W. Yao, M. Li. and B. Ramamurthy, "Design of Sparse Grooming Networks for Transporting Dynamic Multigranularity Sub-wavelength Traffic", 2004.

[7] Osama Awwad, Ala Al-Fuqaha and Ammar Rayes, "Performance of WDM Mesh Networks with Limited Traffic Grooming Resources", IEEE WOCN 2007, Singapore, July 2-4, 2007.

[8] Jun Zhou and Xin Yuan,"A study of dynamic Routing and Wavlength assignment with Imprecise network State information", IEEE,Parallel Processing workshop,2002.

[9] Ashok Kumar Pradhan,S.Barat and tanmay De,"A heuristic approach for multicast Traffic grooming in optical WDM Mesh network",I.J.computer Network and information security,2014.

[10] Amrinder S.Arora and Suresh Subraamaniam,"Converter placement in Wavelength Routing Mesh Topologies" IEEE publication 2000.

[11] Gangxiang shen and RodneyS.Tucker,"Sparse Traffic Grooming in Translucent Optical Networks"IEEE, journal of lightwave technology, VOL. 27, NO. 20, october 15, 2009

[12] H. Zang, J. P. Jue, and B. Mukherjee, "A Review of Routing and Wavelength Assignment Approaches for Wavelength- Routed Optical WDM Networks", SPIE Optical Networks Magazine, vol. 1, no. 1, pp.47-60, January 2000.

[13] Bishwanath Mukherjee, "Optical WDM Networks", Springer Publication 2006.

[14] Keyao Zhu, Hongyue Zhu and Bishwanath Mukherjee, "Traffic Grooming in Optical WDM Mesh Networks", Springer Publication 2006.

[15] Dutta, R,Rouskas, G.N, "Traffic grooming in WDM networks: past and future, Net- work", IEEE , vol.16, no.6, pp. 46- 56, Nov/Dec 2002 